\title{
Interference Management in Cognitive Radio Systems with Feasibility Detection
}

\author{
Sudhir Singh, Member, IEEE, Paul D. Teal, Senior Member, IEEE, Pawel A. Dmochowski, Senior Member, IEEE, \\ and Alan J. Coulson, Senior Member, IEEE
}

\begin{abstract}
In this paper, we consider a cognitive radio system with $N$ secondary user (SU) pairs sharing spectrum with a pair of primary users (PU). The SU power allocation problem is formulated as a capacity maximisation problem under $P U$ and $S U$ quality of service and SU peak power constraints. We show our problem formulation is a geometric program and can be solved with convex optimisation techniques. We examine the effect of PU transmissions in our formulations. Solutions for both lowand high- signal-to-interference-and-noise ratio (SINR) scenarios are provided. We show that including the PU capacity in the optimisation problem in some circumstances leads to increased PU performance while not significantly degrading SU capacity. In a practical wireless communications system, accurate channel state information (CSI) is not often available hence we formulate power allocation problems with both perfect and imperfect CSI and analyse the performance loss incurred due to imperfect CSI. Furthermore, we present a novel method of detecting and removing infeasible $\mathrm{SU}$ quality of service constraints from the $\mathrm{SU}$ power allocation problem that results in considerably improved SU performance. Cumulative distribution functions of capacity for various Rayleigh fading channels are presented.
\end{abstract}

\section{INTRODUCTION}

A large number of papers have appeared on various aspects of cognitive radio (CR) systems, including fundamental information theoretic capacity limits (see, for example, [1-7]). In an underlay CR system the secondary users (SUs) protect the primary user (PU) by regulating their transmit power to maintain the PU receiver interference below a well defined threshold level. The limits on this received interference level at the PU receiver can be imposed by an average/peak constraint [2], or a minimum value for its signal-to-interference-andnoise ratio (SINR) [4]. While imposing an additional channel state information (CSI) requirement [5], the advantage of using an SINR-based PU protection mechanism is that it removes the constant interference threshold, thus benefiting the SUs when the PU link has large SINR.

Power control in conventional wireless networks has been extensively studied in the literature [8-10]. Power control in

Copyright (c) 2013 IEEE. Personal use of this material is permitted. However, permission to use this material for any other purposes must be obtained from the IEEE by sending a request to pubs-permissions@ieee.org.

Sudhir Singh and Alan J. Coulson are with Callaghan Innovation, Lower Hutt, New Zealand (email:\{sudhir.singh,alan.coulson\}@callaghaninnovation.govt.nz)

Paul D. Teal and Pawel. A. Dmochowski are with School of Engineering and Computer Science, Victoria University of Wellington, Wellington, New Zealand (email:\{paul.teal,pawel.dmochowski\}@vuw.ac.nz)

Portions of this work were presented at IEEE International Conference on Communications (ICC) 2012, Ottawa, Canada and Australian Communications Theory Workshop (AusCTW) 2012, Wellington, New Zealand.
CR systems presents its own unique challenges. In spectrum sharing applications, SU's power must be allocated in a manner that achieves the goals of the CR system while not adversely affecting the operation of the PU. Generally the goals of the $\mathrm{CR}$ are not compatible with the goals of the $\mathrm{PU}$; for instance, increasing SU's power to increase SU's capacity will tend to increase interference to the PU. There is a growing body of literature on power control and capacity of CR systems. In [11], soft sensing information was used for optimal power control to maximise capacity of one SU pair coexisting with one PU pair. The impacts of SU's transmission power on the occurrence of spectrum opportunities and the reliability of opportunity detection was analysed in [12]. In [13], dynamic programming was used to develop a power control strategy for one SU pair under a Markov model of the PU's spectrum usage. Optimal power allocation strategies to achieve the ergodic capacity and the outage capacity of one SU pair coexisting with one PU pair under different types of power constraints and fading channel models were obtained in [6]. Power control using game-theoretic approaches has been proposed in $[14,15]$. Power control for CR systems using geometric programming has been proposed in [16-18]. In [17], a CR relay system with one cognitive source, one relay and a cognitive destination coexisting with a PU pair was considered and an optimisation problem to minimise the total CR transmit power under a peak interference constraint was formulated and solved using geometric programming. A minimax approach was used in [18] to minimise the maximum transmit power for a CR system coexisting with a PU-Rx. The interference caused by a PU-Tx to the SU-Rxs was not considered in the problem formulation of [18]. In [16], a distributed approach was used for power allocation to maximise SU sum capacity under a peak interference constraint, but the approach did not include the interference caused by the PU-Tx and the problem was only analysed for a high SINR scenario.

Convex optimisation methods are widely used in the design and analysis of communications systems. Many problems that arise in communications signal processing can be cast or converted into convex optimisation problems which allow analytical or numerical solutions to be calculated easily [19]. In [20], several problems for designing optimal dynamic resource allocation in CR systems are formulated and the key role that convex optimisation plays in finding the optimal solutions is demonstrated.

The problem posed in this paper has been presented in previous works [21] including feasibility detection [22]. This paper improves on the previous results by presenting a solution 
which is robust to channel uncertainties.

The contributions of this paper are as follows.

- We formulate the SU's power allocation problem as a capacity maximisation problem under PU and SU quality of service (QoS) and SU peak power constraints. We show that it can be solved using geometric programming and convex optimisation techniques.

- Unlike in [16-18,20], where the PU's interference at each SU-Rx is neglected, we evaluate the effect of the PU's interference by explicitly including it in our formulations. We present solutions for both low and high SINR scenarios.

- Most of the cognitive radio literature adopts a SU centric view and, apart from guaranteeing minimum QoS to PU, does not consider the PU-SU system as a whole. We show that considering the system capacity in the optimisation problem, in some circumstances, results in improved PU's performance without a significant penalty in SU's capacity. Optimisation strategies for different channel conditions are presented.

- We formulate a robust SU power allocation problem under channel uncertainties by considering a PU outage probability constraint. Through numerical simulations we show that significant losses in SU's performance can be expected when perfect CSI is not available.

- We present a novel method of detecting and removing infeasible SU's QoS constraints from the SU power allocation problem that results in considerably improved SU's performance.

The performance resulting from the optimisation problems outlined above is demonstrated by means of capacity cumulative distribution functions (CDFs) for various channel conditions. Although we only consider flat Rayleigh channels, the framework developed in this paper can be readily extended to other channel models such as Ricean or Nakagami.

The rest of this paper is organised as follows. In Section II, the system model is introduced. Optimum SU power allocation when full CSI is available is discussed in Section III and solutions for both high and low SINR scenarios presented. In Section IV, we present a novel method of detecting and removing infeasible SU's QoS constraints from the SU power allocation problem that results in considerably improved SU's performance. Robust SU power allocation under channel uncertainties is discussed in Section V and in Section VI, we extend feasibility detection to the robust SU power allocation problem. Simulation results are presented in Section VII and conclusions in Section VIII.

\section{SySTEM MODEL}

As shown in Fig. 1, we consider a cognitive radio system with a single $\mathrm{PU}$ and $N \mathrm{SU}$ transmitters communicating simultaneously over a common channel to their respective receivers. Independent, point-to-point, flat Rayleigh fading channels are assumed for all links in the network. Let $g_{\mathrm{p}}=$ $\left|h_{\mathrm{p}}\right|^{2}, g_{\mathrm{ss}}^{(i j)}=\left|h_{\mathrm{ss}}^{(i j)}\right|^{2}, g_{\mathrm{ps}}^{(i)}=\left|h_{\mathrm{ps}}^{(i)}\right|^{2}$ and $g_{\mathrm{sp}}^{(j)}=\left|h_{\mathrm{sp}}^{(j)}\right|^{2}$ denote the instantaneous channel powers of the PU-Tx to PU-Rx, SU-Tx $j$ to SU-Rx $i$, PU-Tx to SU-Rx $i$ and SU-Tx $j$ to

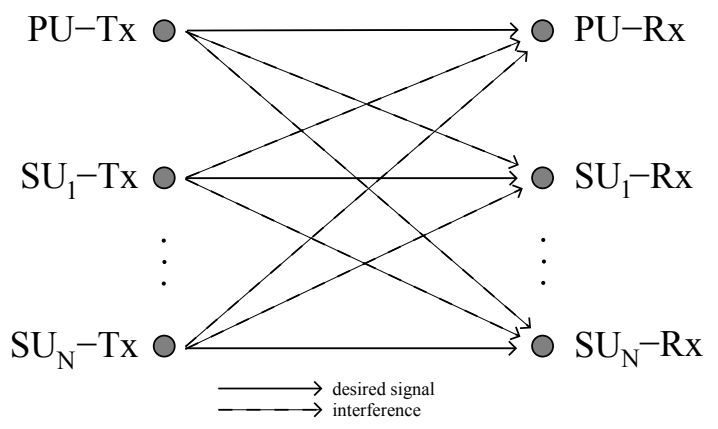

Fig. 1. System Model

PU-Rx links, respectively. For notational convenience we will denote $g_{\mathrm{s}}^{(i)}=g_{s s}^{(i i)}$. Furthermore, we assume that the channel powers for the PU and each of the $N$ SUs are independent and identically distributed (iid) and are governed by their corresponding parameters $\mathbb{E}\left(g_{p}\right)=\Omega_{\mathrm{p}}, \mathbb{E}\left(g_{\mathrm{s}}^{(i)}\right)=\Omega_{\mathrm{s}} \forall i$, $\mathbb{E}\left(g_{\mathrm{ss}}^{(i j)}\right)=\Omega_{\mathrm{ss}} \forall i \neq j, \mathbb{E}\left(g_{\mathrm{ps}}^{(i)}\right)=\Omega_{\mathrm{ps}} \forall i$ and $\mathbb{E}\left(g_{\mathrm{sp}}^{(j)}\right)=\Omega_{\mathrm{sp}}$ $\forall j . \mathbb{E}(\cdot)$ denotes the expectation operator.

In our model the SINR at the $i$ th $\mathrm{SU}$ receiver is given by

$$
\gamma_{\mathrm{s}}^{(i)}=\frac{P_{\mathrm{s}}^{(i)} g_{\mathrm{s}}^{(i)}}{\sum_{j=1, j \neq i}^{N} P_{\mathrm{s}}^{(j)} g_{\mathrm{ss}}^{(i j)}+P_{\mathrm{p}} g_{\mathrm{ps}}^{(i)}+\sigma_{\mathrm{s}}^{2}}
$$

and that at the PU receiver by

$$
\gamma_{\mathrm{p}}=\frac{P_{\mathrm{p}} g_{\mathrm{p}}}{\sum_{j=1}^{N} P_{\mathrm{s}}^{(j)} g_{\mathrm{sp}}^{(j)}+\sigma_{\mathrm{p}}^{2}},
$$

where $P_{\mathrm{s}}^{(j)}$ and $P_{\mathrm{p}}$ are the $j$ th SU and PU transmit powers, respectively, and $\sigma_{\mathrm{s}}^{2}$ and $\sigma_{\mathrm{p}}^{2}$ are the additive white Gaussian noise (AWGN) variance at the $i$ th SU-Rx and PU-Rx, respectively. We also note that that there is a maximum transmit power constraint, $P_{\mathrm{s}, \max }^{(j)}$, on the SU transmitters which may be due either to regulatory or hardware limitations. This is denoted by

$$
P_{\mathrm{s}}^{(j)} \leq P_{\mathrm{s}, \max }^{(j)}
$$

Additionally, the vector $\mathbf{P}_{\mathrm{S}}$ is used to collectively refer to the set of SU transmit powers, i.e., $\mathbf{P}_{\mathrm{s}} \triangleq\left[P_{\mathrm{s}}^{(1)} \ldots P_{\mathrm{s}}^{(N)}\right]^{T}$.

In a cognitive radio system the secondary users are allowed to operate as long as they can guarantee a certain level of quality of service (QoS) to the primary user. Hence, in our analysis we impose an SINR constraint, $\gamma_{T}$, at the PU receiver

$$
\gamma_{\mathrm{p}} \geq \gamma_{\mathrm{T}}
$$

The PU's capacity is given by

$$
C_{\mathrm{p}}=\log _{2}\left(1+\gamma_{\mathrm{p}}\right)
$$

while the SU's sum capacity is denoted by

$$
C_{\Sigma}=\sum_{i=1}^{N} C_{i},
$$


where the individual capacity of the $i$ th $\mathrm{SU}$ is given by

$$
C_{i}=\log _{2}\left(1+\gamma_{\mathrm{s}}^{(i)}\right) .
$$

Using (3) and (4), the system capacity can then be expressed as

$$
C_{\text {sys }}=C_{\mathrm{p}}+C_{\Sigma} .
$$

$C_{\Sigma}$ and $C_{\text {sys }}$ are the performance metrics optimised in Sections III-VI of this paper.

The main system variables can be parameterised as follows. We denote by $c_{1}=\frac{\Omega_{\mathrm{sp}}}{\Omega_{\mathrm{s}}}$ the ratio of interference to desired channel power. Similarly,

$$
c_{2}=\frac{\gamma_{\mathrm{T}}}{P_{\mathrm{p}} \Omega_{\mathrm{p}} / \sigma_{\mathrm{p}}^{2}}
$$

represents the ratio of the minimum target SINR to the mean signal-to-noise ratio (SNR) at the PU-Rx. Hence, increasing $c_{2}$ corresponds to reducing the allowable interference, with the case of $c_{2}=1$ corresponding to zero average allowable interference. Finally, $c_{3}=\frac{\Omega_{\mathrm{ss}}}{\Omega_{\mathrm{s}}}$ parametrises the relative channel power of desired to interfering SU links.

\section{SU POWER OPTIMISATION}

In this section, we aim to find the SU's power allocation such that the SU's sum capacity, $C_{\Sigma}$, or the system capacity, $C_{\text {sys }}$, is maximised while maintaining the PU receiver QoS above the threshold $\gamma_{\mathrm{T}}$, and keeping within the SU's transmit power budget. We may additionally choose to set minimum SINR thresholds, $\gamma_{\mathrm{s}, \min }^{(i)}$ on the $i$ th SU receiver. This represents a practical limitation on SU receivers below which the receivers fail to operate with acceptable performance. We assume that the power allocation problem is solved by a central SU controller and a control channel for the exchange of all necessary information needed for solving the problem exists. Furthermore, we assume that we are unable to control the PU's transmit power and the PU transmits at a constant power of $P_{\mathrm{p}}$. In this section, we formulate the SU power allocation problem under the assumption that perfect CSI for all links are available which allows us to obtain fundamental limits on capacity. However, in practice the channel gains would need to be estimated, hence the capacities obtained in this section provide an upper bound. In Section V, we consider the case when perfect CSI is not available and there is a non-zero probability of PU outage which we constrain. Mathematically we solve the following suite of optimisation problems.

1) SU Capacity Maximisation:

$$
\begin{aligned}
\underset{\mathbf{P}_{\mathrm{s}}}{\operatorname{maximise}} & C_{\Sigma} \\
\text { s.t. } & \gamma_{\mathrm{p}} \geq \gamma_{\mathrm{T}} \\
& P_{\mathrm{s}}^{(j)} \leq P_{\mathrm{s} \text { max }}^{(j)}, \quad j=1, \ldots, N(8 \mathrm{c}) \\
\text { (and o.s.t.) } & \gamma_{\mathrm{s}}^{(i)} \geq \gamma_{\mathrm{s}, \min }^{(i)}, \quad i=1, \ldots, N \text { (8d) }
\end{aligned}
$$

where s.t. and o.s.t. stand for "subject to" and "optionally subject to", respectively. The only difference between problems (8a)-(8c) and (8a)-(8d) is that (8a)-(8d) includes SU QoS constraints, whereas (8a) $-(8 \mathrm{c})$ does not.
2) System Capacity Maximisation:

$$
\begin{aligned}
\underset{\mathbf{P}_{\mathrm{s}}}{\operatorname{maximise}} & C_{\mathrm{sys}} \\
\text { s.t. } & (8 \mathrm{~b}) \text { and }(8 \mathrm{c}) \\
\text { (and o.s.t.) } & (8 \mathrm{~d})
\end{aligned}
$$

From (4) and (5) it is obvious that maximising the objective in (8) is equivalent to maximising $\prod_{i=1}^{N}\left(1+\gamma_{\mathrm{s}}^{(i)}\right)$. Similarly, for (9) we seek to maximise $\left(1+\gamma_{\mathrm{p}}\right) \cdot \prod_{i=1}^{N}\left(1+\gamma_{\mathrm{s}}^{(i)}\right)$. Problems (8) and (9) can be modified to minimisation problems by taking the reciprocal of the objectives. The suite of optimisation problems are nonlinear and non-convex and generally hard to solve [19]. We proceed by dividing our problem into high and low SINR scenarios.

\section{A. High SINR Scenario}

When the SINR at every receiver is high, $C_{\mathrm{p}}, C_{\Sigma}$ and $C_{\text {sys }}$ given in (3)-(6) can be approximated by

$$
\begin{aligned}
C_{\mathrm{p}} & \approx \log _{2}\left(\gamma_{\mathrm{p}}\right) \\
C_{\Sigma} & \approx \log _{2}\left(\prod_{i=1}^{N} \gamma_{\mathrm{s}}^{(i)}\right) \\
C_{\mathrm{sys}} & \approx \log _{2}\left(\gamma_{\mathrm{p}} \cdot \prod_{i=1}^{N} \gamma_{\mathrm{s}}^{(i)}\right) .
\end{aligned}
$$

These approximations are valid when $\gamma_{\mathrm{p}}$ and $\gamma_{\mathrm{s}}^{(i)}$ are much larger than $0 \mathrm{~dB}$, e.g., $10 \mathrm{~dB}$ or more. Using the approximations in (10), the optimisation problems (8) and (9) can be written in minimisation form as

1) High SINR SU Capacity Maximisation :

$$
\begin{aligned}
\underset{\mathbf{P}_{\mathrm{s}}}{\operatorname{minimise}} & \prod_{i=1}^{N}\left(\frac{1}{\gamma_{\mathrm{s}}^{(i)}}\right) \\
\text { s.t. } & (8 \mathrm{~b}),(8 \mathrm{c}) \text { and optionally (8d) }
\end{aligned}
$$

2) High SINR System Capacity Maximisation :

$$
\begin{aligned}
\underset{\mathbf{P}_{\mathrm{s}}}{\operatorname{minimise}} & \left(\frac{1}{\gamma_{\mathrm{p}}}\right) \cdot \prod_{i=1}^{N}\left(\frac{1}{\gamma_{\mathrm{s}}^{(i)}}\right) \\
\text { s.t. } & (8 \mathrm{~b}),(8 \mathrm{c}) \text { and optionally (8d) }
\end{aligned}
$$

Problems (11) and (12) fall into a class of nonlinear, nonconvex optimisation problems known as geometric programs (GP) (see [19,23] for a complete reference on the subject). GP can, however, be transformed to convex optimisation problems and solved efficiently in polynomial time by interior point methods [24].

Through straightforward manipulation of the second and third constraints, problems (11) and (12) can be transformed into a standard form GP [19]. Once in this form, they can be solved to obtain the optimum SU power allocation. The resulting performance is evaluated in Section VII. 


\section{B. Low SINR Scenario}

In the low SINR scenario the approximation (10) is no longer valid and so our capacity maximisation optimisation problems are given by

1) Low SINR SU Capacity Maximisation :

$$
\begin{aligned}
\underset{\mathbf{P}_{\mathrm{s}}}{\operatorname{minimise}} & \prod_{i=1}^{N}\left(\frac{1}{1+\gamma_{\mathrm{s}}^{(i)}}\right) \\
\text { s.t. } & (8 \mathrm{~b}),(8 \mathrm{c}) \text { and optionally (8d) }
\end{aligned}
$$

2) Low SINR System Capacity Maximisation :

$$
\begin{aligned}
\underset{\mathbf{P}_{\mathrm{s}}}{\operatorname{minimise}} & \left(\frac{1}{1+\gamma_{\mathrm{p}}}\right) \cdot \prod_{i=1}^{N}\left(\frac{1}{1+\gamma_{\mathrm{s}}^{(i)}}\right) \\
\text { s.t. } & (8 \mathrm{~b}),(8 \mathrm{c}) \text { and optionally (8d) }
\end{aligned}
$$

The objectives in problems (13) and (14) are ratios of posynomials and hence they are not themselves posynomials. Optimisation problems of this nature are not GP and are known as Complementary GP [23]. Complementary GPs are non-convex problems but can be solved with an iterative technique known as the single condensation method [23]. In each iteration, the feasible point computed in the previous iteration is used to approximate the denominator of the objective monomial. Since a ratio of posynomial and monomial is a posynomial [19], the resulting problem is a GP. The procedure is repeated until the solution converges on an optimum of the original Complementary GP. It should be noted that convergence to a local or global minimum is possible, however, extensive numerical experiments (Section VII) have found empirically that the solution always converges to the global minimum. The posynomial is approximated with a monomial using the geometric-arithmetic mean inequality [23]

$$
\sum_{k} \delta_{k} v_{k} \geq \prod_{k} v_{k}^{\delta_{k}}
$$

where $v_{k} \geq 0, \delta_{k} \geq 0$ and $\sum_{k} \delta_{k}=1$. If we let $u_{k}=\delta_{k} v_{k}$, then (15) can be written as

$$
\sum_{k} u_{k} \geq \prod_{k}\left(\frac{u_{k}}{\delta_{k}}\right)^{\delta_{k}} .
$$

Note that equality in (16) holds when $\delta_{k}=u_{k} / \sum_{k} u_{k}$. The term on the left hand side of (16) resembles the denominator of our objective, i.e. a sum of monomials. Hence, if we let $u_{k}\left(\mathbf{P}_{\mathrm{s}}\right)$ be the monomial terms of the denominator and $\delta_{k}=u_{k}\left(\mathbf{P}_{\mathrm{s}}\right) / \sum_{k} u_{k}\left(\mathbf{P}_{\mathrm{s}}\right)$, then from (16) it is clear that the denominator can be approximated around a feasible $\mathbf{P}_{\mathrm{s}}$ with a product of monomials. Since the approximation is always an under-estimator of the original posynomial, minimising the condensed objective guarantees that the solution moves towards a minimum of the original objective function. An adaptation of a commonly used algorithm [10,23] for solving the low SINR capacity maximisation problem is presented below.

The single condensation method presented above is a general method of solving the power allocation problem and can also be used to solve the high SINR scenario without using the

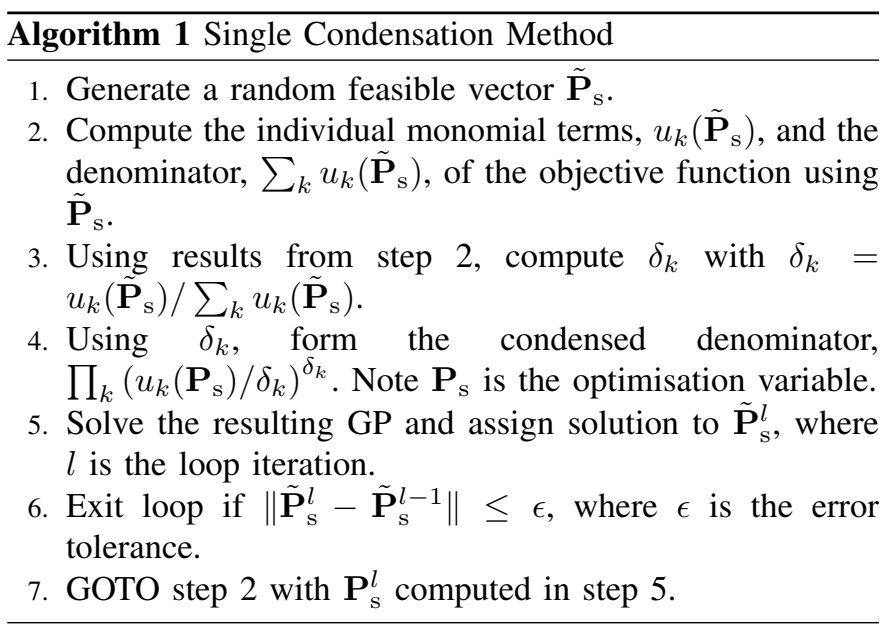

approximation (10) or mixed scenario cases in which some of the receivers in the system have high SINR and others have low SINR.

\section{SU POWER OPTIMISATION WiTh FEASIBILITY DETECTION}

Optimisation problems (8a)-(8d) and (9a)-(9c) are infeasible if any one of the SU QoS constraints is infeasible. This has an adverse effect on capacity since no SUs are able to access the channel if the QoS constraint cannot be met for any one (or more) SU. Thus, one SU which violates the QoS constraint renders the entire optimisation infeasible. Capacity can be improved by selecting the optimum subset of SU's that do not violate the QoS constraints and maximise capacity. However, this selection process is a NP-hard combinatorial optimisation problem which is extremely difficult to solve. A suboptimal method that improves capacity is to exclude the violating SUs from transmission. In this section we formulate a method of detecting and removing the violating SUs from the optimisation problem. The issue of fairness among SUs and the tradeoff between fairness and capacity is beyond the scope of this paper. Our method is based on a feasibility detection technique, known as sum of infeasibilities, that is commonly used in interior point methods to find a strictly feasible starting point [19]. We form the problem

$$
\begin{aligned}
\underset{\mathbf{P}_{\mathrm{s}}, \mathbf{s}}{\operatorname{minimise}} & \mathbf{1}^{\mathrm{T}} \mathbf{s} \\
\text { s.t. } & \frac{\gamma_{\mathrm{T}}}{\gamma_{\mathrm{p}}} \leq \mathbf{s}_{0} \\
& \frac{\gamma_{\mathrm{s}, \min }^{(i)} \leq \mathbf{s}_{i}, \quad i=1, \ldots, N}{\gamma_{\mathrm{s}}^{(i)}} \quad P^{(j)}, \quad j=1, \ldots, N \\
& P_{\mathrm{s}}^{(j)} \leq P_{\mathrm{s}, \text { max }}, \quad \\
& \mathbf{s} \succeq \mathbf{1},
\end{aligned}
$$

where 1 is a vector of length $N+1$ with all entries equal to one, $\mathbf{s} \in \mathbb{R}_{++}^{N+1}$ and $\succeq$ is the elementwise greater than or equal to comparison operator. For fixed $\mathbf{P}_{\mathrm{s}}$, the optimal values of $\mathbf{s}_{0}$ and $\mathbf{s}_{i}$ are $\max \left(\gamma_{\mathrm{T}} / \gamma_{\mathrm{p}}, 1\right)$ and $\max \left(\gamma_{\mathrm{s}, \min }^{(i)} / \gamma_{\mathrm{s}}^{(i)}, 1\right)$ respectively, so in problem (17), we are minimising the sum of the infeasibilities. The optimum value of (17) is $N+1$ and 
achieved if and only if the constraints (8b), (8c) and (8d) are feasible. It follows that all feasible SU QoS constraints will have the corresponding element in the vector $\mathbf{s}$ equal to one.

Let $\mathcal{I}$ represent the set of feasible SU QoS constraints determined from the solution of problem (17). We then solve the following SU power optimisation problems in which the violating SUs are removed

1) SU Capacity Maximisation with Feasible SU QoS Constraints:

$$
\begin{aligned}
\underset{\mathbf{P}_{\mathrm{s}}}{\operatorname{maximise}} & C_{\Sigma} \\
\text { s.t. } & \gamma_{\mathrm{p}} \geq \gamma_{\mathrm{T}} \\
& \gamma_{\mathrm{s}}^{(i)} \geq \gamma_{\mathrm{s}, \text { min }}^{(i)}, \quad i \in \mathcal{I} \\
& P_{\mathrm{s}}^{(j)} \leq P_{\mathrm{s}, \text { max }}^{(j)}, \quad j \in \mathcal{I}
\end{aligned}
$$

2) System Capacity Maximisation with Feasible SU QoS Constraints:

$$
\begin{aligned}
\underset{\mathbf{P}_{\mathrm{s}}}{\operatorname{maximise}} & C_{\mathrm{sys}} \\
\text { s.t. } & (18 \mathrm{~b}),(18 \mathrm{c}) \text { and }(18 \mathrm{~d})
\end{aligned}
$$

Problems (18) and (19) can be solved using methods presented in Section III for the low and high SINR scenarios.

\section{SU POWER OPTIMISATION UNDER ChanNel UNCERTAINTIES}

So far we have assumed that perfect CSI of all links are available. However, in practise this assumption may not be valid. For our analysis, we assume that the channel between the SU-Txs and SU-Rxs are accurately known through the SU's channel estimation procedure and those between the PU transmitter and SU receivers can be accurately measured, for example through knowledge of the PU's pilot symbols. As stated in Section II, the PU-Tx to PU-Rx and the $j$ th SU-Tx to PU-Rx channel gains are iid and for the analysis of this section, we assume that only the mean channel gains, $\Omega_{\mathrm{p}}$ and $\Omega_{\mathrm{sp}}$, of these links are known, i.e., the instantaneous values of $g_{\mathrm{p}}$ and $g_{\mathrm{sp}}^{(j)}$ are not known. In this section we consider the $\mathrm{SU}$ power optimisation problem under these uncertainties.

In our formulation we consider the PU's outage probability as a QoS parameter. In the system under consideration, outage occurs when the PU's SINR, $\gamma_{\mathrm{p}}$, falls below the PU's SINR threshold, $\gamma_{\mathrm{T}}$. The outage probability is expressed as

$$
\begin{aligned}
P_{\mathrm{o}} & =\operatorname{Prob}\left(\gamma_{\mathrm{p}} \leq \gamma_{\mathrm{T}}\right) \\
& =\operatorname{Prob}\left(P_{\mathrm{p}} g_{\mathrm{p}} \leq \gamma_{\mathrm{T}}\left(\sum_{j=1}^{N} P_{\mathrm{s}}^{(j)} g_{\mathrm{sp}}^{(j)}+\sigma_{\mathrm{p}}^{2}\right)\right) .
\end{aligned}
$$

In a Rayleigh fading environment, $g_{\mathrm{p}}$ and $g_{\mathrm{sp}}^{(j)}$ are exponentially distributed random variables with means $\Omega_{\mathrm{p}}$ and $\Omega_{\mathrm{sp}}$ respectively. Under these conditions, the outage probability is commonly known to have the following form (see, for example, [25])

$$
\mathrm{P}_{\mathrm{o}}=1-e^{-c_{2}} \prod_{j=1}^{N}\left(\frac{1}{1+\frac{\gamma_{\mathrm{T}} P_{\mathrm{s}}^{(j)} \Omega_{\mathrm{sp}}}{P_{\mathrm{p}} \Omega_{\mathrm{p}}}}\right),
$$

where $c_{2}$ is given by (7).

To formulate the SU power optimisation problem under channel uncertainty, we replace the PU's SINR threshold in problems (8) and (9) by the outage probability constraint. Furthermore, since instantaneous CSI for PU-Tx to PU-Rx and SU-Txs to PU-Rx links are not available, in the optimisation problem that seeks to maximise the system capacity-(9)the PU's SINR is calculated using the expected values of these links i.e., $g_{p}$ and $g_{\mathrm{sp}}^{(j)}$ in (2) are replaced with $\Omega_{\mathrm{p}}$ and $\Omega_{\mathrm{sp}}$ respectively. Hence, given a maximum allowable outage probability, $\mathrm{P}_{\mathrm{o}, \max }$, the optimisation problems are expressed as:

1) SU Capacity Maximisation Under Channel Uncertainty:

$$
\begin{aligned}
\underset{\mathbf{P}_{\mathrm{s}}}{\operatorname{maximise}} & C_{\Sigma} \\
\text { s.t. } & \prod_{j=1}^{N}\left(1+\frac{\gamma_{\mathrm{T}} P_{\mathrm{s}}^{(j)} \Omega_{\mathrm{sp}}}{P_{\mathrm{p}} \Omega_{\mathrm{p}}}\right) \\
& \leq \frac{e^{-c_{2}}}{1-\mathrm{P}_{\mathrm{o}, \max }}
\end{aligned}
$$

(and o.s.t.)

2) System Capacity Maximisation Under Channel Uncertainty:

$$
\begin{aligned}
\underset{\mathbf{P}_{\mathrm{s}}}{\operatorname{maximise}} & C_{\mathrm{sys}} \\
\text { s.t. } & (8 \mathrm{c}) \text { and }(21 \mathrm{~b}) \\
\text { (and o.s.t.) } & (8 \mathrm{~d})
\end{aligned}
$$

We have only shown the general formulations here. However, using the approximations in Section III-A, capacity maximisation problems for high SINR scenario can be constructed. Problems (21) and (22) can be solved using techniques described in Sections III-A and III-B.

\section{SU POWER OPTIMISATION With FEASIBILITY Detection Under Channel Uncertainties}

The feasibility detection technique formulated in Section IV can be incorporated into the formulations developed in Section V to improve the SU's performance when SU's QoS constraints are imposed. We form the problem

$$
\begin{aligned}
\underset{\mathbf{P}_{\mathrm{s}}, \mathbf{s}}{\operatorname{minimise}} & \mathbf{1}^{\mathrm{T}} \mathbf{s} \\
\text { s.t. } & \left(\frac{1-\mathrm{P}_{\mathrm{o}, \text { max }}}{e^{-c_{2}}}\right) \\
& \cdot \prod_{j=1}^{N}\left(1+\frac{\gamma_{\mathrm{T}} P_{\mathrm{s}}^{(j)} \Omega_{\mathrm{sp}}}{P_{\mathrm{p}} \Omega_{\mathrm{p}}}\right) \leq \mathbf{s}_{0} \\
& \frac{\gamma_{\mathrm{s}, \min }^{(i)} \leq \mathbf{s}_{i}, \quad i=1, \ldots, N}{\gamma_{\mathrm{s}}^{(i)}} \\
& P_{\mathrm{s}}^{(j)} \leq P_{\mathrm{s}, \max }^{(j)}, \quad j=1, \ldots, N \\
& \mathbf{s} \succeq \mathbf{1},
\end{aligned}
$$




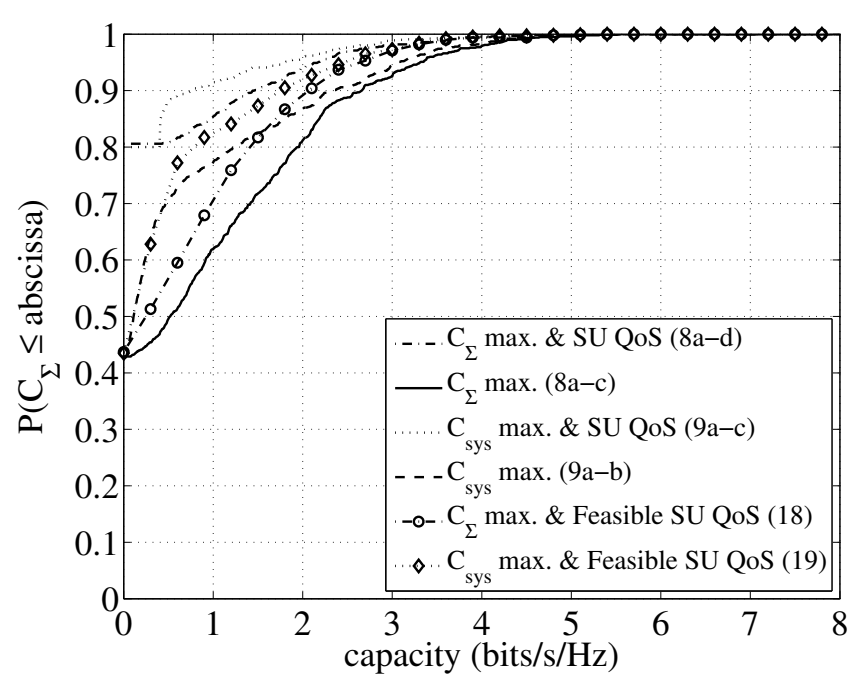

Fig. 2. $C_{\Sigma}$ CDF with perfect CSI for Scenario $A, \gamma_{\mathrm{T}}=5 \mathrm{~dB}$. The same legend is used in Figs. 3-7.

the solution of which gives us the set $\mathcal{I}$, the set of feasible SU QoS constraints. As in Section IV, we then solve the following problems in which the violating SUs are removed

1) SU Capacity Maximisation Under Channel Uncertainty with Feasible SU QoS Constraints:

$$
\begin{aligned}
\underset{\mathbf{P}_{\mathrm{s}}}{\operatorname{maximise}} & C_{\Sigma} \\
\text { s.t. } & (21 \mathrm{~b}) \\
& \gamma_{\mathrm{s}}^{(i)} \geq \gamma_{\mathrm{s}, \text { min }}^{(i)}, \quad i \in \mathcal{I} \\
& P_{\mathrm{s}}^{(j)} \leq P_{\mathrm{s}, \max }^{(j)}, \quad j \in \mathcal{I}
\end{aligned}
$$

2) System Capacity Maximisation Under Channel Uncertainty with Feasible SU QoS Constraints:

$$
\begin{aligned}
\underset{\mathbf{P}_{\mathrm{s}}}{\operatorname{maximise}} & C_{\mathrm{sys}} \\
\text { s.t. } & (21 \mathrm{~b}),(24 \mathrm{c}) \text { and }(24 \mathrm{~d})
\end{aligned}
$$

Problems (24) and (25) can be solved using the methods presented in Section III for the low and high SINR scenarios.

\section{Simulation Results ANd Discussion}

We now present the results of simulations that require solution of the optimisation problems formulated in this paper, specifically evaluating the CDFs of the resulting capacities. We consider a system with $N=3$ SUs. In all simulations we have set $P_{\mathrm{p}}=0 \mathrm{dBw}, P_{\mathrm{s}, \text { max }}^{(i)}=0 \mathrm{dBw}, \sigma_{\mathrm{p}}^{2}=\sigma_{\mathrm{s}}^{2}=-37 \mathrm{dBw}$ and $\Omega_{\mathrm{p}}=\Omega_{\mathrm{s}}=5 \mathrm{~dB}$. This choice of parameter values allows us to contrast the optimisation problems formulated in this paper in the channel scenarios given below. Simulations for optimisation problems that impose $\mathrm{SU}$ QoS requirements have $\gamma_{\mathrm{s}, \text { min }}^{(i)}=-10 \mathrm{~dB}, i=1, \ldots, N$. In SU power optimisation problems under channel uncertainties we have set the outage probability, $\mathrm{P}_{\mathrm{o}, \max }$, to $5 \%$. We consider the following three channel scenarios

1) Scenario A: High Interference In this scenario $c_{1}=c_{3}=0.9$ which corresponds to each receiver being approximately the same distance

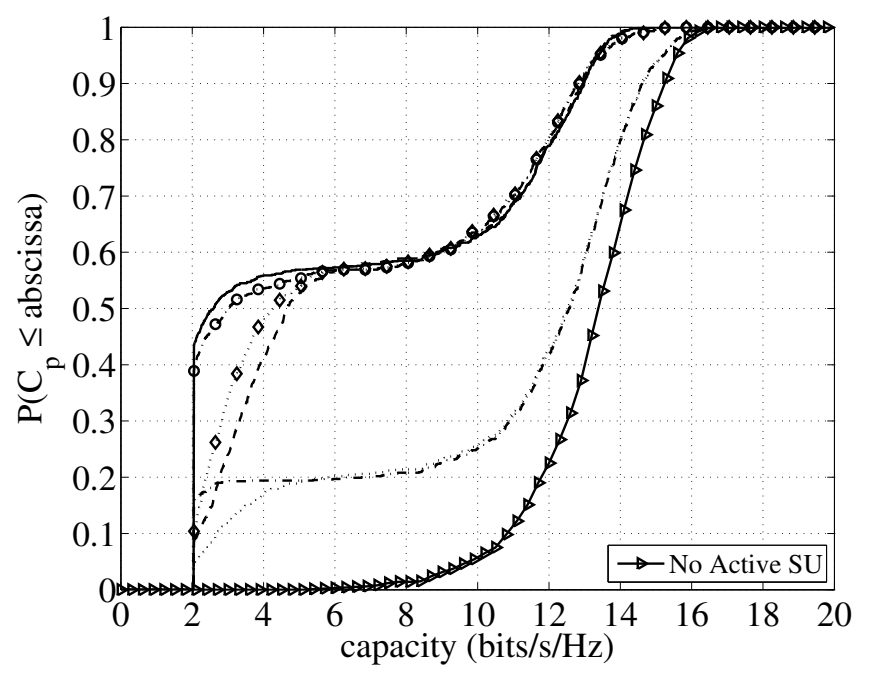

Fig. 3. $C_{\mathrm{P}}$ CDF with perfect CSI for Scenario A, $\gamma_{\mathrm{T}}=5 \mathrm{~dB}$.

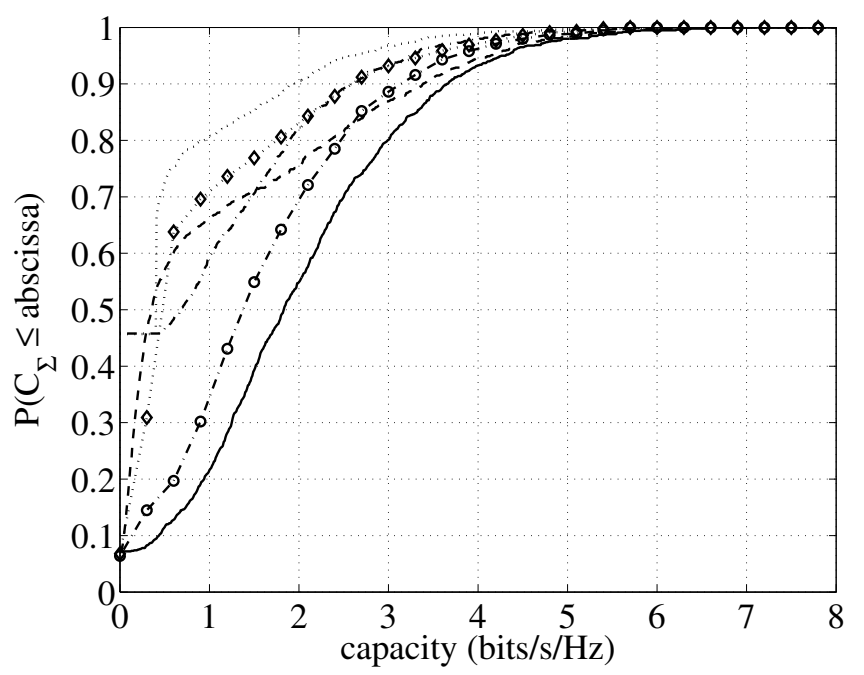

Fig. 4. $C_{\Sigma}$ CDF with perfect CSI for Scenario $B, \gamma_{\mathrm{T}}=5 \mathrm{~dB}$.

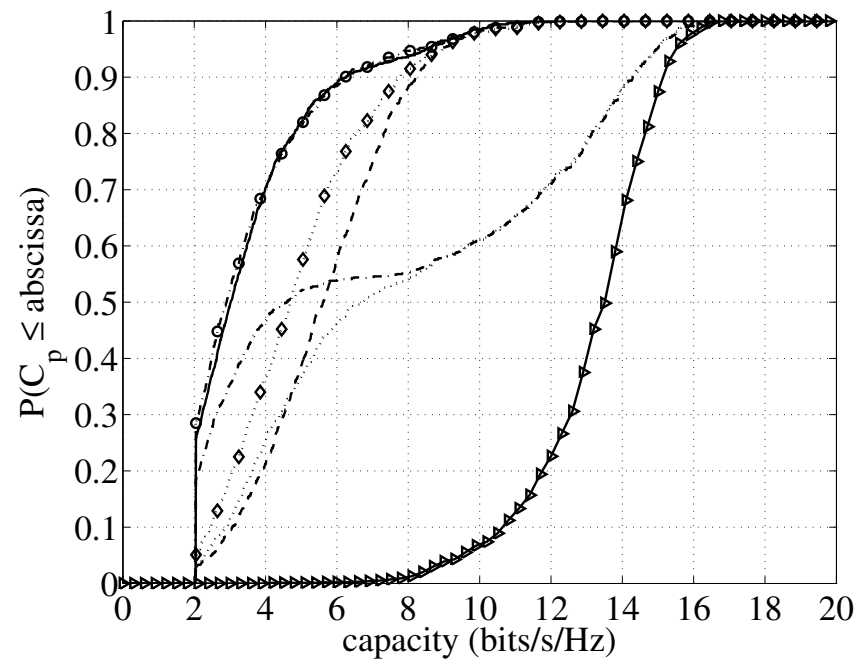

Fig. 5. $C_{\mathrm{P}}$ CDF with perfect CSI for Scenario $B, \gamma_{\mathrm{T}}=5 \mathrm{~dB}$. 


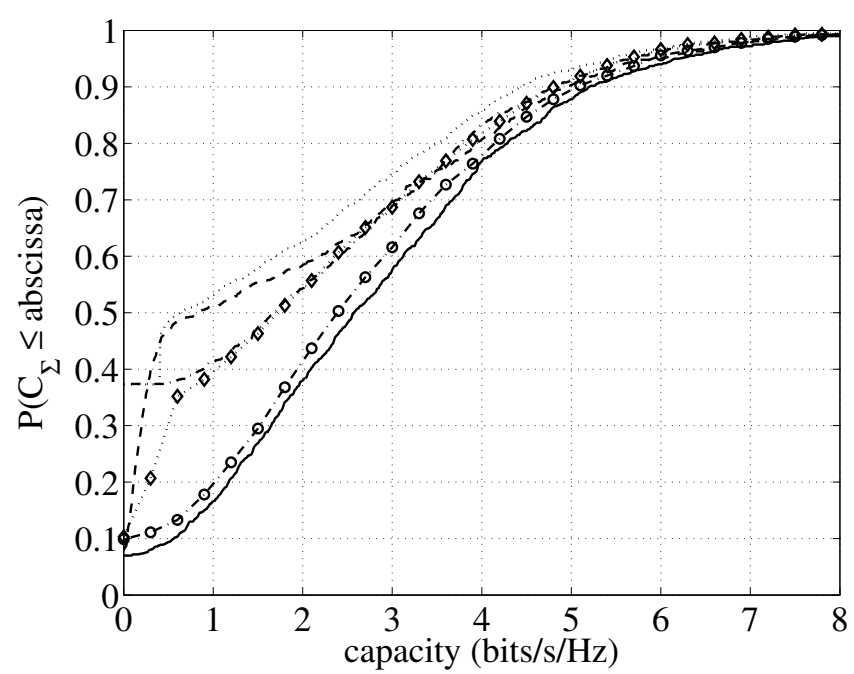

Fig. 6. $C_{\Sigma} \mathrm{CDF}$ with perfect CSI for Scenario $C, \gamma_{\mathrm{T}}=5 \mathrm{~dB}$.

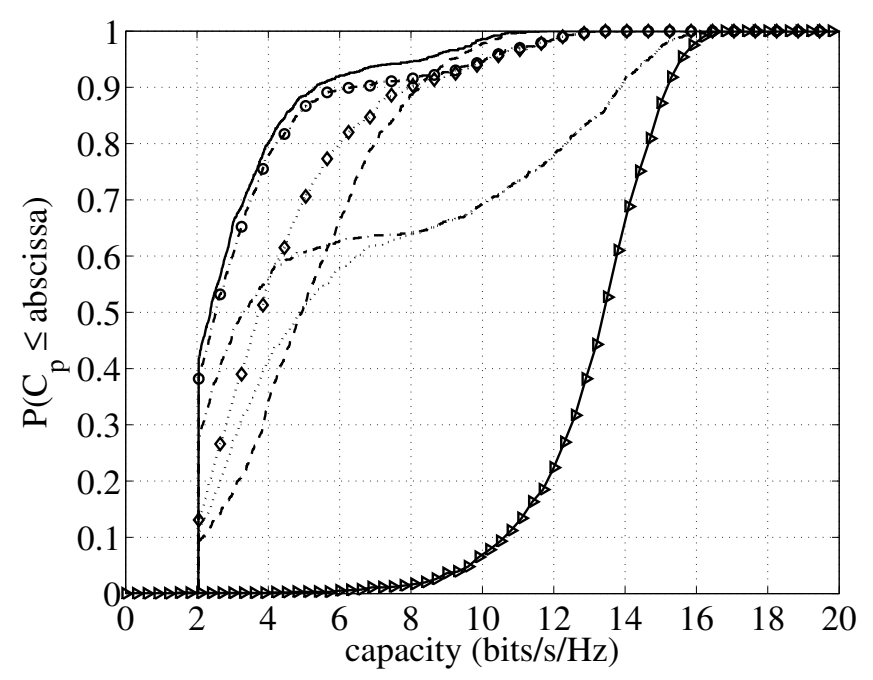

Fig. 7. $C_{\mathrm{P}}$ CDF with perfect CSI for Scenario $C, \gamma_{\mathrm{T}}=5 \mathrm{~dB}$.

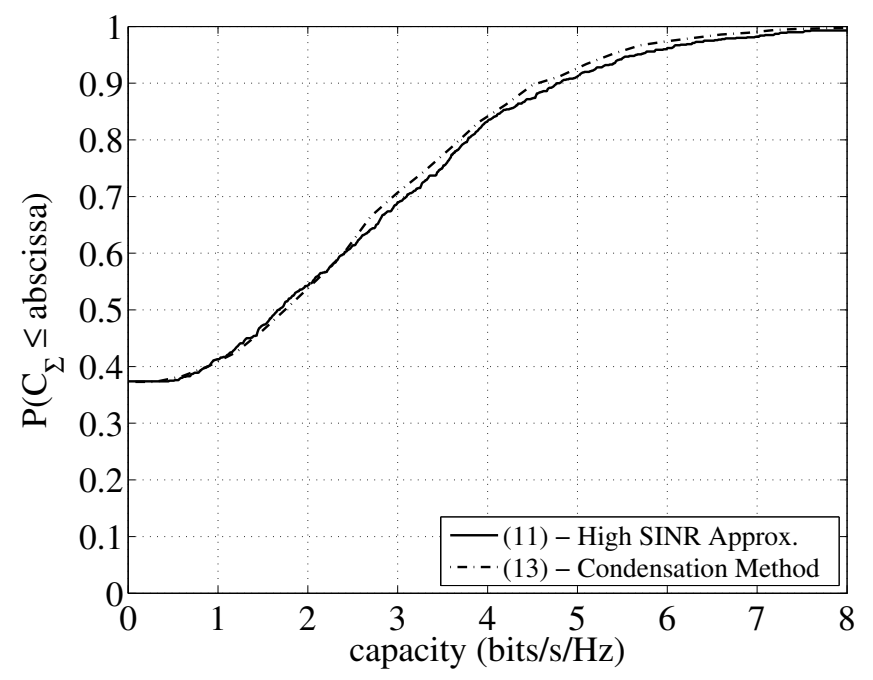

Fig. 8. $C_{\Sigma} \mathrm{CDF}$ (SU QoS imposed) comparison of high SINR approximation and condensation method for Scenario $C, \gamma_{\mathrm{T}}=5 \mathrm{~dB}$.

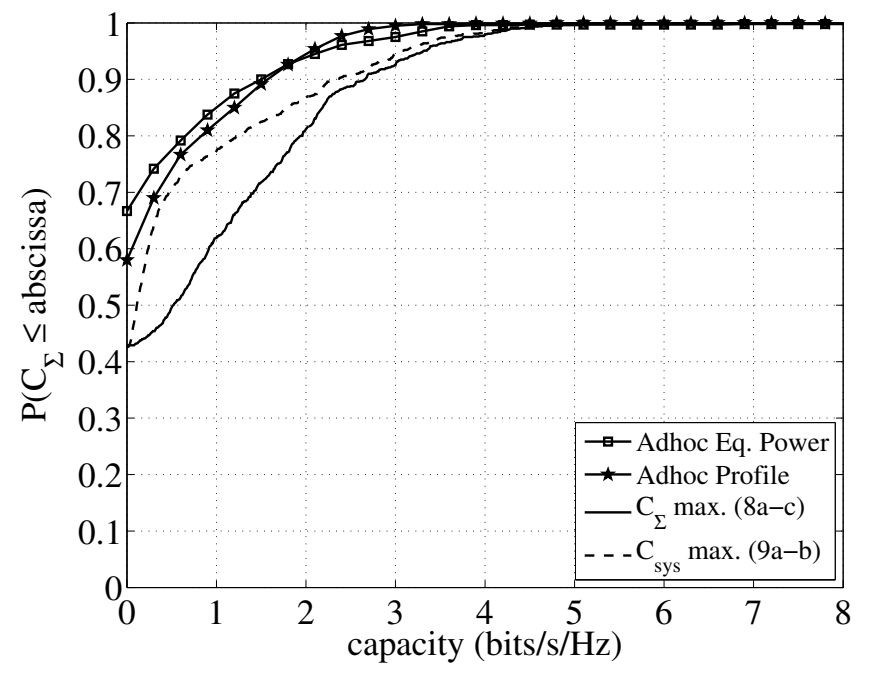

Fig. 9. $C_{\Sigma} \mathrm{CDF}$ comparison of proposed method and ad hoc methods for Scenario $A, \gamma_{\mathrm{T}}=5 \mathrm{~dB}$.

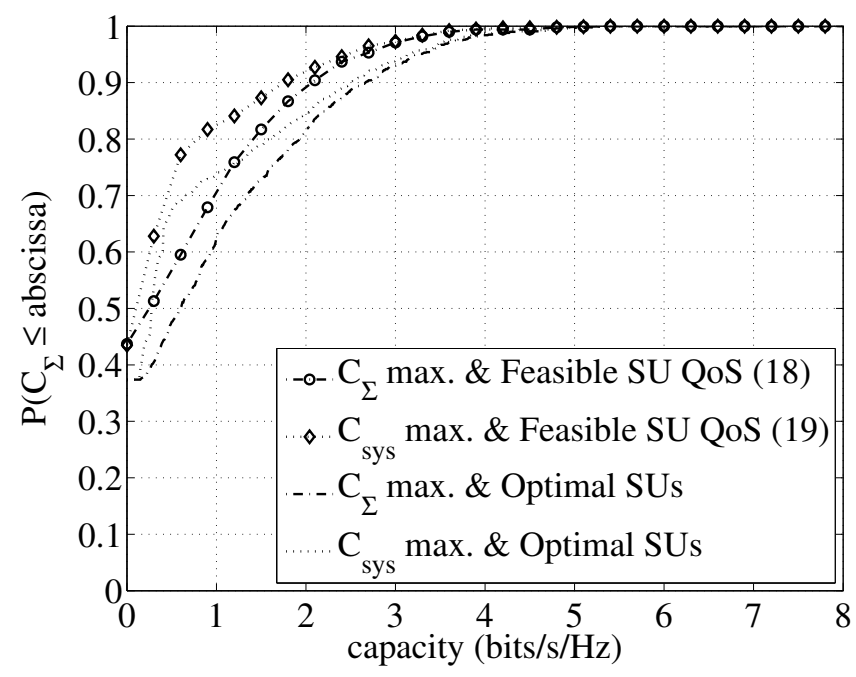

Fig. 10. $C_{\Sigma}$ CDF comparison of proposed feasibility detection and brute force methods for Scenario A, $\gamma_{\mathrm{T}}=5 \mathrm{~dB}$.

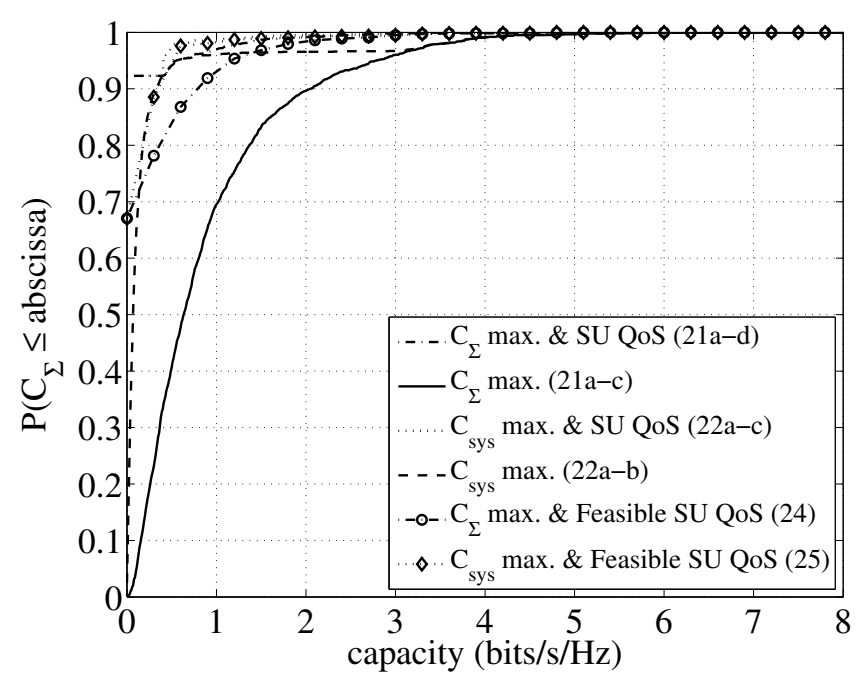

Fig. 11. $C_{\Sigma}$ CDF under channel uncertainties for Scenario $B, \gamma_{\mathrm{T}}=5 \mathrm{~dB}$, $\mathrm{P}_{\mathrm{o}, \max }=5 \%$. The same legend is used in Fig. 12. 


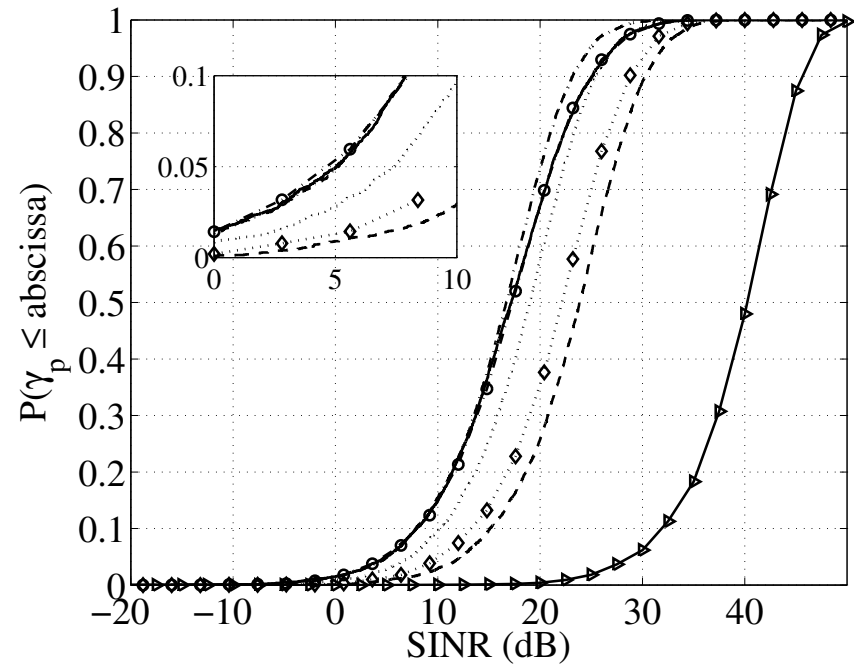

Fig. 12. $\gamma_{\mathrm{p}}$ CDF under channel uncertainties for Scenario $B, \gamma_{\mathrm{T}}=5 \mathrm{~dB}$, $\mathrm{P}_{\mathrm{o}, \max }=5 \%$.

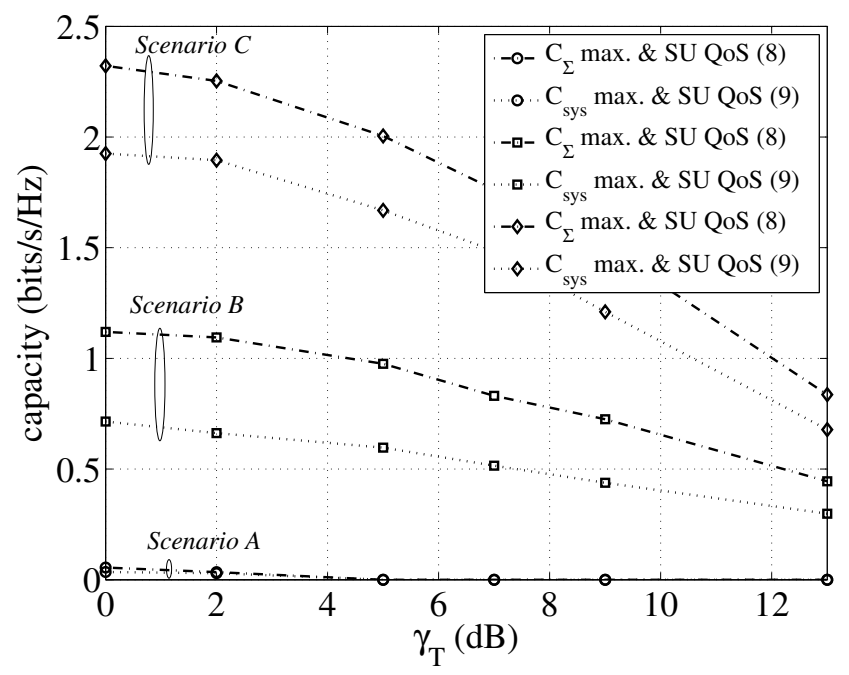

Fig. 13. Mean $C_{\Sigma}$ when SU QoS are imposed as a function of $\gamma_{\mathrm{T}}$ with perfect CSI.

from all transmitters. This results in high interference among all users, thus making the PU's QoS constraint difficult to satisfy. The SINR is expected to be low, hence we use the low SINR method of Section III-B to obtain the solution.

2) Scenario B: Low PU and High SU Interference

In this scenario $c_{1}=0.1$ and $c_{3}=0.9$. Here, the PU experiences low interference from the SUs since it is approximately 3 times (assuming $1 / d^{2}$ path loss) further away from SU-Txs than the PU-Tx. As a result, the PU's QoS constraint is easily satisfied. However, SU to SU interference is very prominent. In this scenario, the SINR at the SUs will be low and therefore we obtain the solution using the low SINR method of Section III-B.

3) Scenario $C$ : Low Interference

In this scenario $c_{1}=c_{3}=0.1$ which corresponds to each receiver being approximately 3 times further away

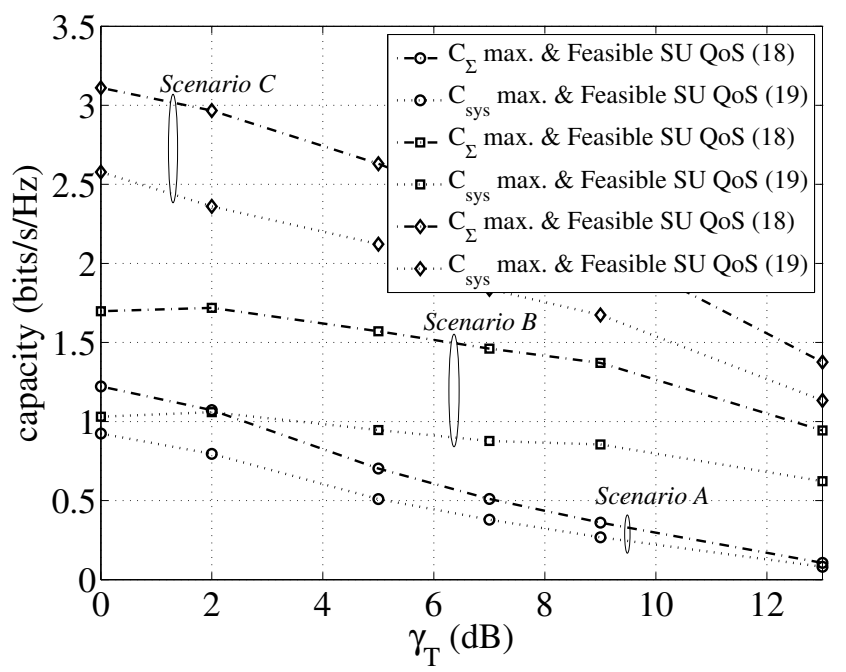

Fig. 14. Mean $C_{\Sigma}$ as a function of $\gamma_{\mathrm{T}}$ with perfect CSI and feasibility detection.

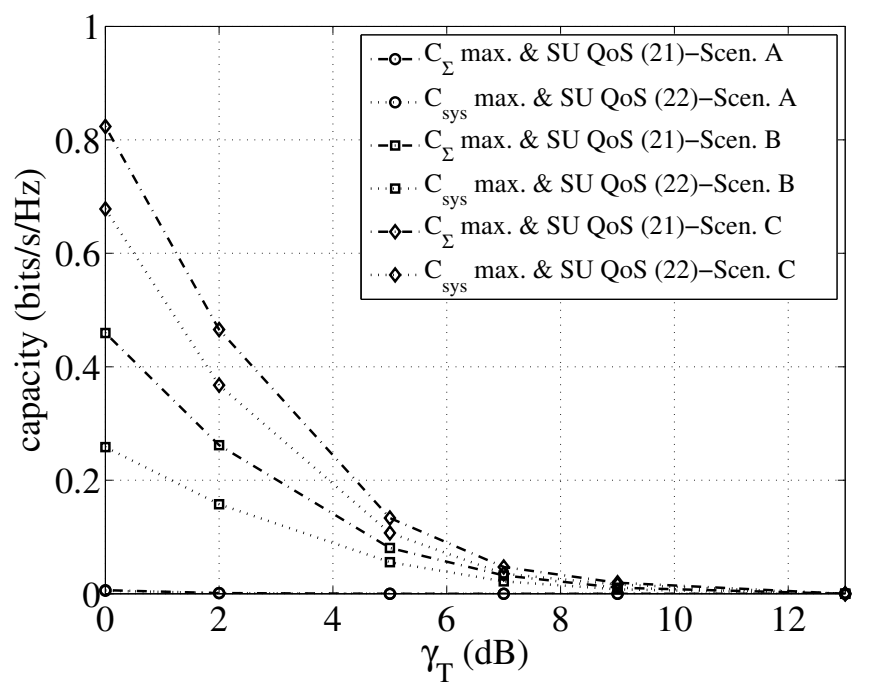

Fig. 15. Mean $C_{\Sigma}$ when SU QoS are imposed as a function of $\gamma_{\mathrm{T}}$ under channel uncertainties, $\mathrm{P}_{\mathrm{o}, \max }=5 \%$.

from the interfering transmitters than its own transmitter. This results in low interference between all users, thus making the PU's QoS constraint easy to satisfy. Since the SINR at all receivers is expected to be high, this scenario is solved using the high SINR method of Section III-A.

When solving using the low SINR (single condensation) method, each instantiation of the problem is solved multiple times using different random but feasible staring points, $\tilde{\mathbf{P}}_{\mathrm{s}}$, and solutions checked to confirm convergence to the same point. If different solutions are obtained then this indicates convergence to local minima, but in our extensive numerical experiments we have never observed this behaviour.

For our discussion, we define SU blocking probability as the probability that $C_{\Sigma}=0$, i.e, no SUs are able to access the channel.

Results of our proposed methods are compared against the equal power allocation method and a power profile method 


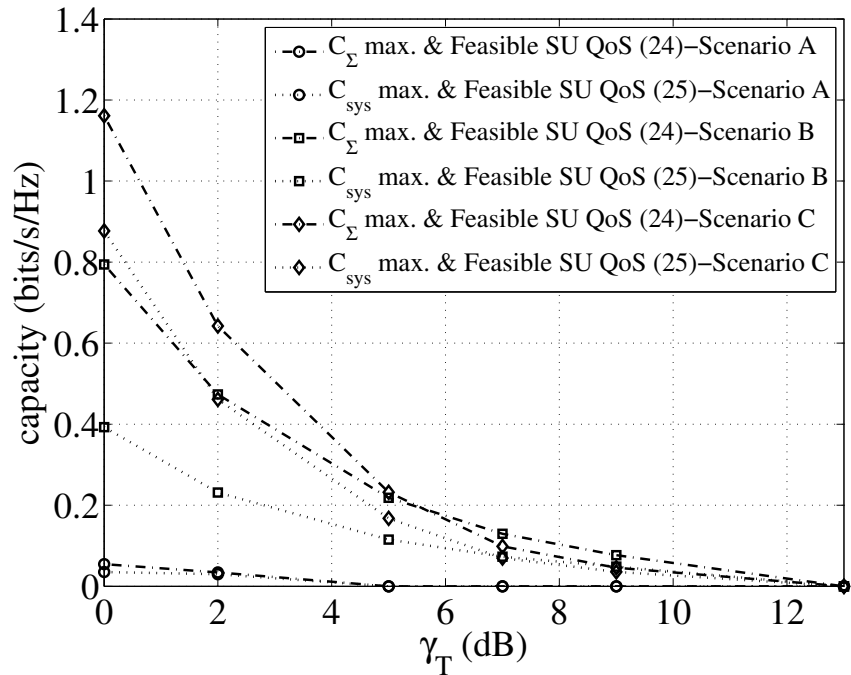

Fig. 16. Mean $C_{\Sigma}$ as a function of $\gamma_{\mathrm{T}}$ under channel uncertainties and feasibility detection, $\mathrm{P}_{\mathrm{O}, \max }=5 \%$

analogous to the "poor man's waterfilling" method [26] where we allocate power proportionally to $g_{\mathrm{s}}^{(i)} / g_{\mathrm{sp}}^{(i)}$. We refer to these methods as ad hoc allocation methods. Note that the $a d$ hoc allocation methods do not impose a minimum SU QoS requirement, hence a fair comparison is only possible against problems (8a)-(8c) and (9a-9b).

We also compare our proposed feasibility detection method against the brute force method of selecting the optimum set of SU transmitters. The brute force method performs an exhaustive search and finds the set of SUs that achieve the highest capacity.

Figs. 2-7 show $C_{\Sigma}$ and $C_{\mathrm{p}}$ capacity CDFs for the three channel conditions with $\gamma_{\mathrm{T}}=5 \mathrm{~dB}$ when perfect CSI is available. The legends of Figs. 2 and 3 are applicable for all 6 of these figures. From Figs. 2, 4 and 6, we observe that in all three scenarios, problem (8a)-(8c) performs the best in terms of SU performance. All optimisation problems result in non-zero SU blocking probability due to either PU's QoS constraints or both PU's and SU's QoS constraints. It is evident that feasibility detection results in a significant improvement in SU's performance in all channel scenarios, for instance, in Scenario $A$ the SU blocking probability (Fig. 2) is reduced from $80 \%$ to approximately $42 \%$. Figs. 3, 5 and 7 show the PU's capacity CDF for the three scenarios along with the $\mathrm{CDF}$ for the reference case when no SUs are transmitting. The discontinuity in the graphs correspond to the point at which the optimisation problems become feasible and SU transmissions start. The effect of the SU's transmissions on the PU's capacity is clearly visible.

By noting the effects of including the PU's capacity in the optimisation problems we are able to contrast two optimisation strategies using problems (8a)-(8c) and (9a)-(9b). In Scenario $A$, we observe that the two optimisation problems result in similar median SU sum capacities, however, problem (9a)(9b) results in a much improved median PU's capacity. Hence, when the PU experiences high interference, problem (9a)-(9b) is the better option as it results in improved PU's performance while not adversely affecting the SU's performance. On the other hand, in Scenarios $B$ and $C$, where there is low interference to the PU, using problem (9a)-(9b) improves the PU's performance but adversely affects the SU's performance. Therefore, from the SU's point of view, problem (8a)-(8c) is the preferred option.

As stated previously, Scenario $C$ is solved using the high SINR approximation method. It is also possible to solve it using the more general condensation method. In Fig. 8 we compare the solution of problem (8a)-(8d) obtained using the two methods. From the results, it is evident that the high SINR approximation provides a fairly accurate approximation of the problem.

In Fig. 9 we compare the results of our proposed optimisation problems (8a)-(8c) and (9a-9b) against the ad hoc power allocation methods of [26]. We see that the ad hoc allocation methods are outperformed by the methods proposed in this paper. We have only shown results for Scenario A since similar results are obtained for Scenarios $B$ and $C$.

In Fig. 10 we compare the results of our feasibility detection for Scenario $A$ against the brute force method of selecting the optimum set of SUs. As expected, the brute force method outperforms the proposed method, however, the performance improvement comes at a price of greatly increased computational complexity. Although suboptimal, the proposed feasibility detection method is an efficient method of improving the SU performance.

Figs. 11 and 12 show $C_{\Sigma}$ and $C_{\mathrm{p}}$ capacity CDFs for Scenario $B$ with $\gamma_{\mathrm{T}}=5 \mathrm{~dB}$ when channel uncertainties exist. The legend of Fig. 11 is applicable to Fig. 12. Due to space constraints we only show results for Scenario B. Scenario $C$ results are similar to Scenario B. Results for Scenario A are not shown because, due to unfavourable channel conditions, the PU's outage probability constraint is never satisfied and no SUs are able to access the channel, i.e, there is a $100 \%$ SU blocking probability for all optimisation problems. From Fig. 11, it is clear that there is a significant loss in the SU's performance compared to when perfect CSI is available, for instance, the median $C_{\Sigma}$ obtained using problem (21a)-(21c) is less than half of that obtained using problem (8a)-(8c). Feasibility detection is again seen to reduce the SU blocking probability. Fig. 12 shows the distribution of $\gamma_{p}$ under channel uncertainties. The inset shows the section between $0 \mathrm{~dB}-10 \mathrm{~dB}$ in greater detail and confirms that all optimisation problems attain an outage probability (probability PU's SINR is below $5 \mathrm{~dB}$ ) of $5 \%$ or less. From the SU's point of view, when channel uncertainties exist, problems (24) and (21a)-(21c) are the preferred options depending on whether the SU's QoS constraints are required or not.

Figs. 13-16 plot the mean $C_{\Sigma}$ as a function of $\gamma_{\mathrm{T}}$ for problems that impose SU's QoS constraints. From Figs. 13 and 14 it can be seen that feasibility detection significantly improves the SU's performance in all three scenarios when accurate CSI is available. Fig. 16 shows that when perfect CSI is not available, feasibility detection results in significant improvement of the SU's performance for Scenarios $B$ and $C$ while minor gains for lower values of $\gamma_{\mathrm{T}}$ are seen for Scenario A. Figs. 15 and 16 highlight the fact that unavailability of 
accurate CSI adversely affects the SU's performance, where practically no SU communications are possible in a high interference scenario. This can be improved if the PU relaxes its QoS requirements, either through a reduction of $\gamma_{\mathrm{T}}$ or an increase of $\mathrm{P}_{\mathrm{o}, \max }$ or a combination of both. As expected, accurate knowledge of PU-Tx to PU-Rx and SU-Txs to PURx links are crucial to the SU's performance and large SU's performance losses are expected if accurate CSI of these links are not available.

\section{CONCLUSIONS}

In this paper, we have formulated the SU power allocation problem in a $\mathrm{CR}$ system as a geometric program and obtained capacity CDFs in various channel conditions. We have included the effect of the PU's transmission in our formulations and studied the problem in both high and low SINR scenarios. It has been demonstrated that considering the system capacity in the optimisation problem, in some circumstances, results in improved PU performance without a significant penalty in the SU's capacity. Optimisation strategies for different channel conditions have been presented. Furthermore, we have presented a novel method of detecting and removing infeasible SU's QoS constraints from the SU power allocation problem that results in considerably improved SU's performance. A robust SU power allocation problem under channel uncertainties by considering a PU outage probability constraint has been presented. The results quantify the intuitive importance of PU-Tx to PU-Rx and SU-Txs to PU-Rx CSI and large SU performance losses are expected if accurate CSI of these links are not available.

\section{REFERENCES}

[1] S. A. Jafar and S. Srinivasa, "Capacity limits of cognitive radio with distributed and dynamic spectral activity," IEEE J. Sel. Areas Commun., vol. 25, pp. 529-537, April 2007.

[2] A. Ghasemi and E. S. Sousa, "Fundamental limits of spectrum-sharing in fading environments," IEEE Trans. Wireless Commun., vol. 6, pp. 649-658, February 2007.

[3] L. Musavian and S. Aissa, "Fundamental capacity limits of spectrumsharing channels with imperfect feedback," in Proc. IEEE GLOBECOM 2007, November 2007, pp. 1385-1389.

[4] P. A. Dmochowski, H. A. Suraweera, P. J. Smith, and M. Shafi, "Impact of channel knowledge on cognitive radio system capacity," in Proc. IEEE VTC2010-Fall, September 2010, pp. 1-5.

[5] M. Shafi, H. A. Suraweera, P. J. Smith, and M. Faulkner, "Capacity limits and performance analysis of cognitive radio with imperfect channel knowledge," IEEE Trans. on Veh. Technol., January 2010.

[6] X. Kang, Y.-C. Liang, A. Nallanathan, H. K. Garg, and R. Zhang, "Optimal power allocation for fading channels in cognitive radio networks: Ergodic capacity and outage capacity," IEEE Trans. Wireless Commun., vol. 8, pp. 940-950, February 2009.

[7] C.-X. Wang, X. Hong, H.-H. Chen, and J. Thompson, "On capacity of cognitive radio networks with average interference power constraints," IEEE Trans. Wireless Commun., vol. 8, pp. 1620-1625, April 2009.

[8] C. Sung and W. Wong, "Power control and rate management for wireless multimedia cdma systems," IEEE Trans. Commun., vol. 49, no. 7, pp. $1215-1226$, July 2001.

[9] P. Gupta and P. R. Kumar, "The capacity of wireless networks," IEEE Trans. Inf. Theory, vol. 46, no. 2, pp. 388-404, March 2000.

[10] M. Chiang, C. W. Tan, D. Palomar, D. O'Neill, and D. Julian, "Power control by geometric programming," IEEE Trans. Wireless Commun., vol. 6, no. 7, pp. 2640-2651, July 2007.

[11] S. Srinivasa and S. A. Jafar, "Soft sensing and optimal power control for cognitive radio," IEEE Trans. on Wireless Commun., vol. 9, no. 12, pp. 3638-3649, December 2010.
[12] W. Ren, Q. Zhao, and A. Swami, "Power control in cognitive radio networks: How to cross a multi-lane highway," IEEE J. Sel. Areas Commun., vol. 27, no. 7, pp. 1283-1296, September 2009.

[13] Y. Chen, G. Yu, , Z. Zhang, H. Chen, and P. Qiu, "On cognitive radio networks with opportunistic power control strategies in fading channels," IEEE Trans. Wireless Commun., vol. 7, no. 7, pp. 2752-2761, July 2008.

[14] M. Alayesh and N. Ghani, "Game-theoretic approach for primarysecondary user power control under fast flat fading channels," IEEE Commun. Letters, vol. 15, no. 5, pp. 491-493, May 2011.

[15] P. Setoodeh and S. Haykin, "Robust transmit power control for cognitive radio," Proceedings of the IEEE, vol. 97, no. 5, pp. 915-939, May 2009.

[16] Q. Jin, D. Yuan, and Z. Guan, "Distributed geometric-programmingbased power control in cellular cognitive radio networks," in Proc. VTC 2009, April 2009, pp. 1-5.

[17] D. Li and X. Dai, "Power control in cooperative cognitive radio networks by geometric programming," in Proc. APCC 2009, October 2009, pp. $118-121$.

[18] L. Tang, H. Wang, and Q. Chen, "Power allocation with min-max fairness for cognitive radio networks," in Proc. WCNIS 2010, June 2010, pp. 478-482.

[19] S. Boyd and L. Vandenberghe, Convex Optimization. Cambridge University Press, 2009.

[20] R. Zhang, Y.-C. Liang, and S. Cui, "Dynamic resource allocation in cognitive radio networks: A convex optimization perspective," arXiv, vol. abs/1001.3187, 2010.

[21] S. Singh, P. D. Teal, P. A. Dmochowski, and A. J. Coulson, "Interference management in cognitive radio systems - a convex optimisation approach," in Proc. ICC 2012, June 2012, pp. 1908-1913.

[22] , "Power allocation in underlay cognitive radio systems with feasibility detection," in Proc. AusCTW 2012, February 2012, pp. 135139.

[23] C. S. Beightler and D. T. Philips, Applied Geometric Programming. Wiley, 1976.

[24] Y. Nesterov and A. Nemirovski, Interior Point Polynomial Methods in Convex Programming. SIAM Press, 1994.

[25] S. Kandukuri and S. Boyd, "Optimal power control in interference limited fading wireless channels with outage probability specifications," IEEE Trans. Wireless Commun., vol. 1, no. 1, pp. 46-55, January 2002.

[26] P. Smith, T. King, L. Garth, and M. Dohler, "A power scaling analysis of norm-based antenna selection techniques," IEEE Trans. on Wireless Commun., vol. 7, no. 8, pp. 3140-3149, August 2008. 